\title{
140. Localization of Calcium-Accumulating Structures in the Anterior Byssal Retractor Miuscle of \\ Mytilus edulis and Their Role in Excitation-Contraction Coupling
}

\author{
By Haruo SugI*) and Saoko Atsumi \\ Departments of Physiology and Anatomy, Faculty of Medicine \\ University of Tokyo
}

(Comm. by Yasuji KatsukI, M. J. A., Oct. 12, 1973)

In a previous paper, ${ }^{5)}$ it has been shown that, when external $\mathrm{Ca}$ ions are removed in the absence or with reduced concentrations of $\mathrm{Mg}$ ions, marked reversible contractures are produced in the anterior byssal retractor muscle (ABRM) of Mytilus edulis, and the development of contracture tension was associated with repetitive electrical activity of the fibre membrane. These results are explained by assuming the intracellularly stored $\mathrm{Ca}$ ions, which are located at or near the fibre membrane so that they are released during membrane activity to cause contraction. The present paper deals with electron microscopic determination of localization of the stored $\mathrm{Ca}$ in the ABRM, using pyroantimonate which is known to penetrate intact cell membrane in the presence of osmium to produce electron-opaque precipitate with intracellular cations. ${ }^{3), 4)}$

The ABRM was teased to obtain a fibre bundle of about $1 \mathrm{~mm}$ diameter. The fibre bundle was mounted horizontally in a chamber filled with the experimental solution, and attached to a strain gauge to record isometric tension At the end of experiments, the fibres were fixed by replacing the experimental solution with a $1 \% \mathrm{OsO}_{4}$ solution ( $\mathrm{pH}$ 6.0-6.2 by $0.01 \mathrm{~N}$ acetic acid) containing $2 \%$ potassium pyroantimonate $\left(\mathrm{K}\left[\mathrm{Sb}(\mathrm{OH})_{6}\right]\right)$. Then the fibres were dehydrated in ethanol and embedded in Epon 812. Sections were cut on a PorterBlum ultramicrotome and examined with a Hitachi HU-12 electron microscope unstained or stained with uranyl acetate and lead citrate.

The ABRM fibres, which had been completely relaxed in the standard solution containing $20 \mathrm{mM} \mathrm{Ca}^{5 \text { ) }}$ before fixation, showed three distinct sites of pyroantimonate precipitate deposition (Fig. 1a), namely, (1) at the plasma membrane, (2) at the vesicles subjacent to the fibre surface and (3) at the mitochondria also beneath the fibre

*) Present address: Department of Physiology, School of Medicine, Teikyo University, Itabashi-ku, Tokyo. 


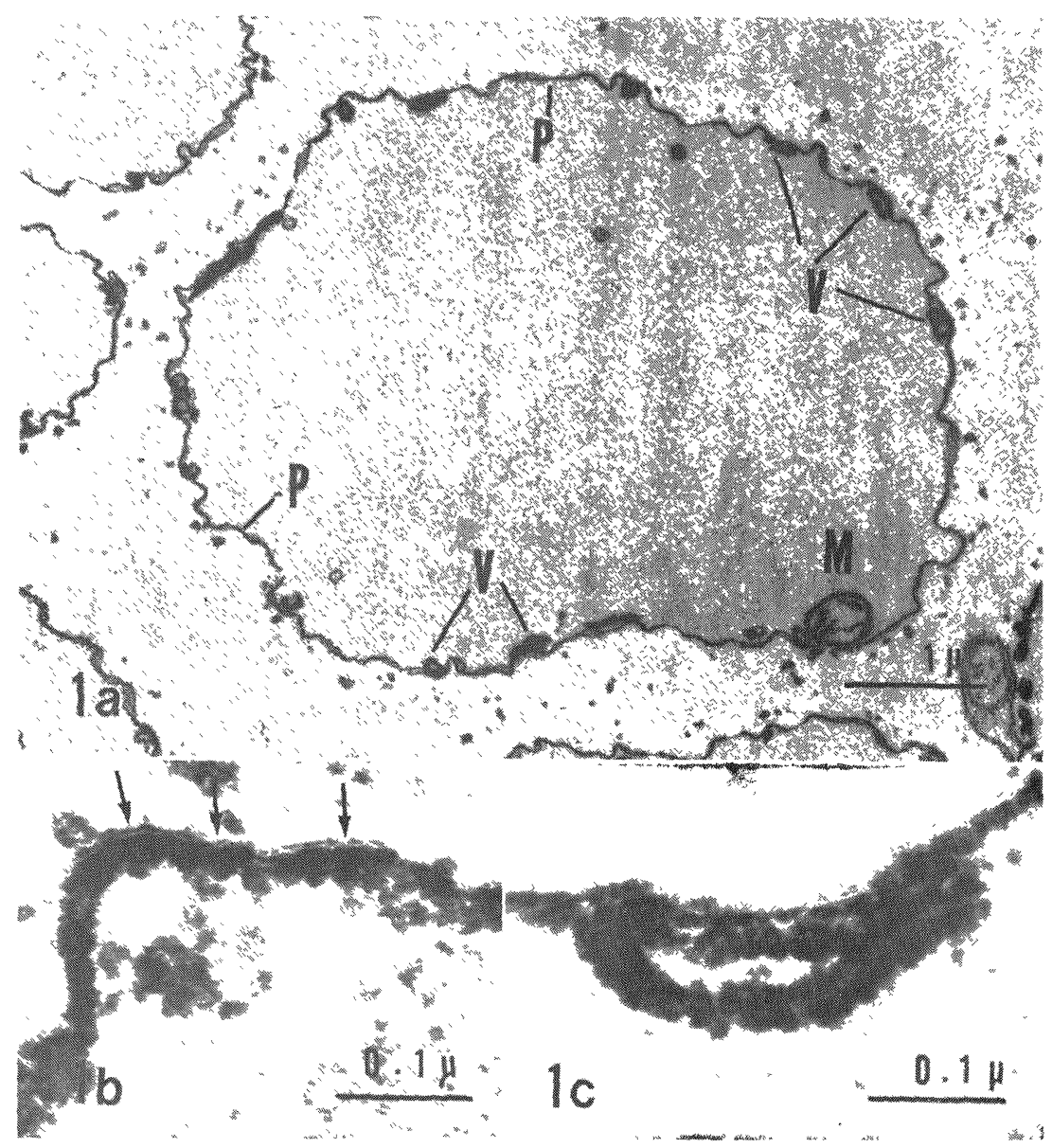

Fig. 1. (a) Cross-section of pyroantimonate-osmium fixed resting ABRM, showing electron-opaque precipitate at the plasma membrane $(P)$, at the vesicles (V) and at the mitochondria (M). Unstained. (b) Higher magnification of fibre surface, showing the localization of the precipitate along the inner surface (inner leaflet) of the plasma membrane. Arrows indicate the outer surface of the plasma membrane. Stained with lead cit. rate and uranyl acetate. (c) Higher magnification of a heavy deposition of pyroantimonate at the vesicle. Note that deposits occur along both inner and outer layers of the unit membrane, while the middle lamina is nonreactive. Unstained.

surface. Closer observation with higher magnifications indicated that the precipitate at the plasma membrane was located only along its inner surface, while the precipitate was seen along both sides of the membrane and in the lumen of the vesicles (Fig. 1b, c). The amount of the precipitate at these sites decreased when the fibres were perfused in a Ca-free solution containing $0.5 \mathrm{mM}$ EGTA for more than $30 \mathrm{~min}$ before fixation. The precipitate still remained if the fibres were 


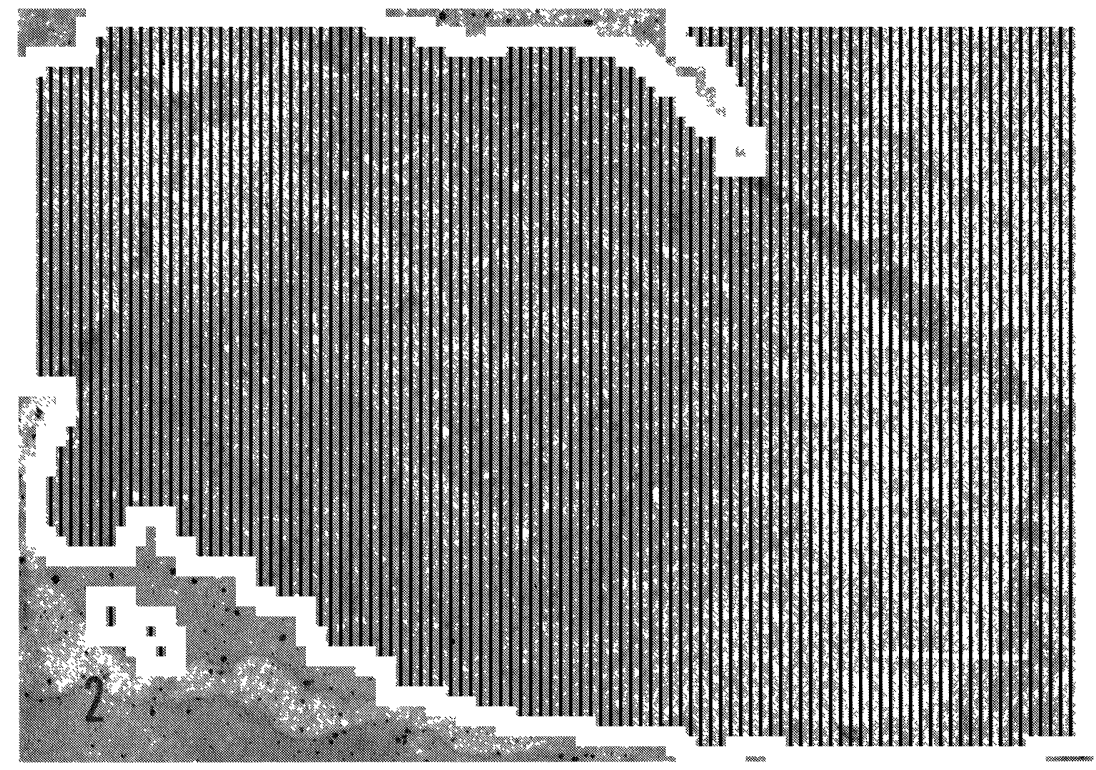

Fig. 2. Fibres fixed during the plateau of Ca-removal contracture. Note the diffuse distribution of the precipitate in the myoplasm in the form of small particles. Unstained.

soaked in a Na-free solution ( $\mathrm{NaCl}$ replaced by sucrose) for $30 \mathrm{~min}$ or more. Examination of the sections with an energy dispersive Xray analyser (JEOL JEM 100B-ASID with EDAX analyser) demonstrated the presence of $\mathrm{Ca}$ and $\mathrm{Sb}$ in the precipitate, but not the presence of other cations including $\mathrm{Na}$ and $\mathrm{Mg}$, indicating that calcium pyroantimonate may form the electron-opaque precipitate.

When the ABRM fibres were made to contract by the application of a Ca-free solution containing $0.5 \mathrm{mM}$ EDTA or EGTA and zero $\mathrm{Mg}$ ions, $\left.{ }^{5}\right)$ and fixed during the plateau of the resulting Ca-removal contracture, an almost complete dispersal of the precipitate into the myoplasm was found to take place (Fig. 2). Furthermore, if the fibres were fixed during the period of spontaneous relaxation, the dispersed precipitate was observed to reaccumulate at the original sites of localization (Fig. 3 ).

The present findings may be taken to indicate that, in the resting ABRM, intracellular Ca ions are accumulated along the inner surface of the fibre membrane, at the vesicles and at the mitochondria, and that the Ca ions may be released from these structures into the myoplasm at least in the case of Ca-removal contractures. Reaccumulation of the released $\mathrm{Ca}$ during the period of spontaneous relaxation may be due to inactivation of the Ca-releasing mechanism during maintained depolarization ${ }^{5)}$ as supposed for spontaneous relaxation 

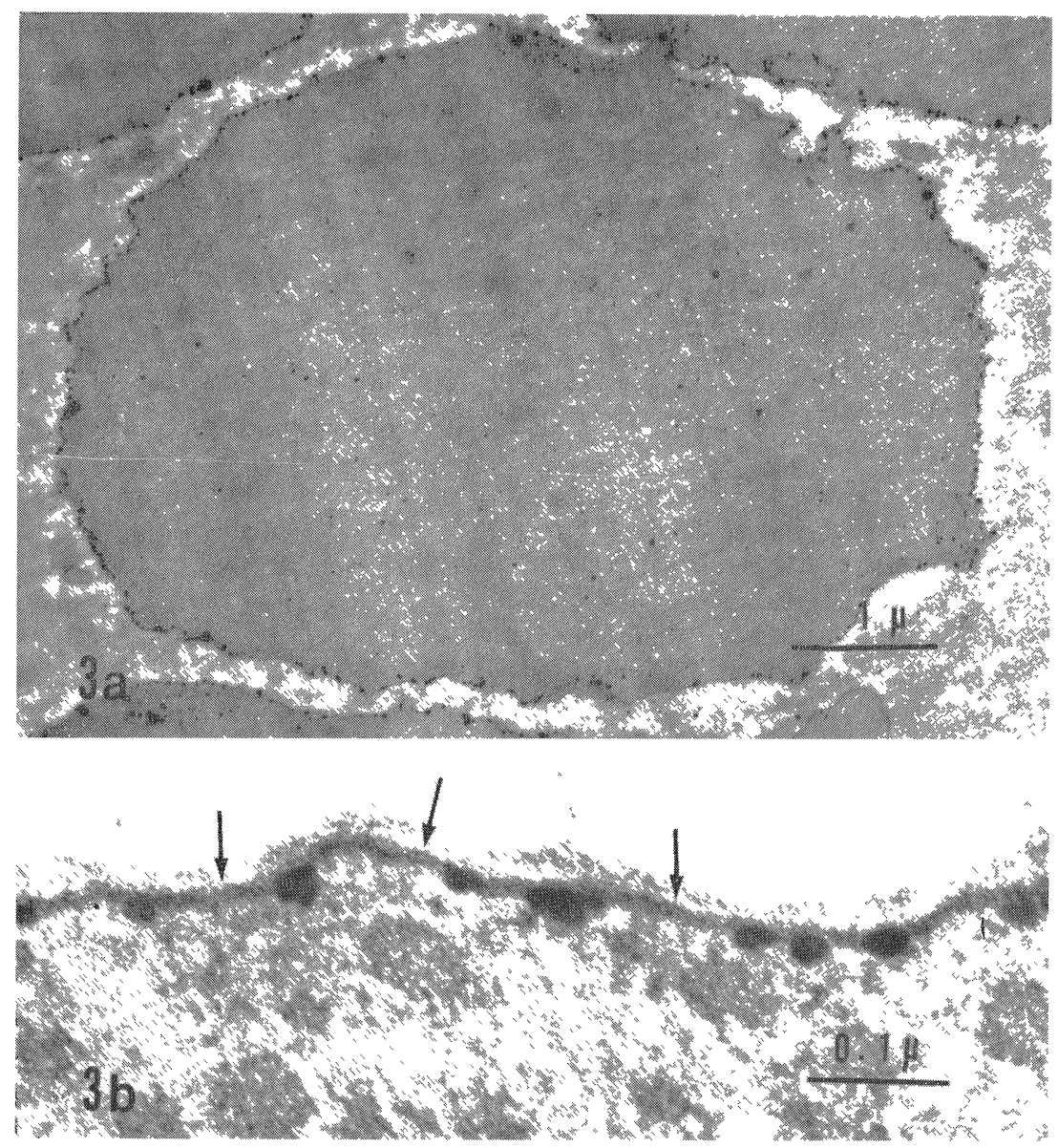

Fig. 3. Fibres fixed during spontaneous relaxation of Ca-removal contracture. (a) The precipitate is again seen along the plasma membrane. (b) Higher magnification of fibre surface shows that the precipitate is located along the inner surface of the plasma membrane. Arrows indicate the outer surface of the plasma membrane. Stained with lead citrate and uranyl acetate.

of K-contractures in frog muscle fibres.2) Heumann" reported that, when the ABRM was perfused with a solution containing calcium, oxalate and ATP, electron-opaque calcium oxalate was seen in the vesicles but not at the plasma membrane. It may be that, in his work, the Ca-accumulating ability of the fibre membrane had been impaired at the time of fixation because of the pretreatment of the fibres with glycerine.

It is of interest to examine the behaviour of the intracellularly stored Ca during active contraction or catch state induced by acetylcholine which is believed to be the neural transmitter substance in the 
ABRM. ${ }^{6}$ ) Some experiments have already been made, and it appears that the stored $\mathrm{Ca}$ is also released into the myoplasm during contractures by acetylcholine, though the results are not yet conclusive. Details of the present work will be published elsewhere with more experimental results.

Acknowledgement. We wish to thank Prof. E. Yamada and Dr. $\mathrm{H}$. Ishikawa for their interest and advice. Our thanks are also due to Miss M. Higuchi for her technical assistance.

\section{References}

1) Heumann, H. (1969): Protoplasma, 67, 111.

2) Hodgkin, A. L., and Horowicz, P. (1960): J. Physiol., 153, 386.

3) Komnick, H., and Komnick, U. (1963) : Z. Zellforsch. Mikrosk. Anat., 60, 12.

4) Legato, M. J., and Langer, G. A. (1969) : J. Cell Biol., 41, 401.

5) Sugi, H. (1971) : Proc. Japan Acad., 47, 683.

6) Twarog, B. M. (1960) : J. Gen. Physiol., 50, 157. 